\title{
Available potential energy density for a multicomponent Boussinesq fluid with arbitrary nonlinear equation of state
}

Article

Accepted Version

Tailleux, R. (2013) Available potential energy density for a multicomponent Boussinesq fluid with arbitrary nonlinear equation of state. Journal of Fluid Mechanics, 735. pp. 499518. ISSN 0022-1120 doi:

https://doi.org/10.1017/jfm.2013.509 Available at https://centaur.reading.ac.uk/34396/

It is advisable to refer to the publisher's version if you intend to cite from the work. See Guidance on citing.

To link to this article DOI: http://dx.doi.org/10.1017/jfm.2013.509

Publisher: Cambridge University Press

All outputs in CentAUR are protected by Intellectual Property Rights law, including copyright law. Copyright and IPR is retained by the creators or other copyright holders. Terms and conditions for use of this material are defined in the End User Agreement.

www.reading.ac.uk/centaur 
Central Archive at the University of Reading

Reading's research outputs online 


\title{
Available potential energy density for a multicomponent Boussinesq fluid with arbitrary nonlinear equation of state
}

\author{
By REMI TAILL E U $\mathbf{X}^{1} \dagger$ \\ ${ }^{1}$ Dept. of Meteorology, University of Reading, Earley Gate, PO Box 243, Reading, RG6 6BB
}

(Received ?? and in revised form ??)

In this paper, the concept of available potential energy (APE) density is extended to a multicomponent Boussinesq fluid with a nonlinear equation of state. As shown by previous studies, the APE density is naturally interpreted as the work against buoyancy forces that a parcel needs to perform to move from a notional reference position at which its buoyancy vanishes to its actual position; because buoyancy can be defined relative to an arbitrary reference state, so can APE density. The concept of APE density is therefore best viewed as defining a class of locally defined energy quantities, each tied to a different reference state, rather than as a single energy variable. An important result, for which a new proof is given, is that the volume integrated APE density always exceeds Lorenz's globally defined APE, except when the reference state coincides with Lorenz's adiabatically re-arranged reference state of minimum potential energy. A parcel reference position is systematically defined as a level of neutral buoyancy (LNB): depending on the nature of the fluid and on how the reference state is defined, a parcel may have one, none, or multiple LNB within the fluid. Multiple LNB are only possible for a multicomponent fluid whose density depends on pressure. When no LNB exists within the fluid, a parcel reference position is assigned at the minimum or maximum geopotential height. The class of APE densities thus defined admits local and global balance equations, which all exhibit a conversion with kinetic energy, a production term by boundary buoyancy fluxes, and a dissipation term by internal diffusive effects. Different reference states alter the partition between APE production and dissipation, but neither affect the net conversion between kinetic energy and APE, nor the difference between APE production and dissipation. We argue that the possibility of constructing APE-like budgets based on reference states other than Lorenz's reference state is more important than has been previously assumed, and we illustrate the feasibility of doing so in the context of an idealised and realistic oceanic example, using as reference states one with constant density and another one defined as the horizontal mean density field; in the latter case, the resulting APE density is found to be a reasonable approximation of the APE density constructed from Lorenz's reference state, while being computationally cheaper.

\section{Introduction}

Margules (1903) and Lorenz (1955) recognised early on that only a fraction - depending on particular circumstances, e.g., stratification, rotation, presence of a background mean flow, whether processes are diabatic or adiabatic - of the total potential energy of

$\dagger$ Present address: Dept. of Meteorology, University of Reading, Earley Gate, Reading, RG6 6BB, United Kingdom 
a given stratified fluid is in general available for conversions with kinetic energy. The concept of available potential energy (APE) formalises such an idea. The problem of deriving explicit theoretical expressions for the APE as a function of particular circumstances remains poorly understood but in the simplest cases. In the literature, the concept of APE is often identified with Lorenz (1955) APE, viz.,

$$
\mathrm{APE}_{\text {Lorenz }}=\int_{V} \rho\left(\mathrm{pe}-\mathrm{pe}_{r}^{*}\right) \mathrm{d} V=\mathrm{PE}-\mathrm{PE}_{r}^{*},
$$

where $\mathrm{PE}$ is the total potential energy of the actual state, while $\mathrm{PE}_{r}^{*}$ is the total potential energy of the reference state of minimum potential energy that can be obtained from an adiabatic re-arrangement of mass (pe and $\mathrm{pe}_{r}^{*}$ are the corresponding specific potential energies). Physically, Lorenz's APE is commonly interpreted as the total amount of potential energy that could in principle be released into kinetic energy in a notional thought experiment in which the actual state would be allowed to relax towards a state of rest by means of a purely adiabatic process. It is useful to distinguish, however, between the general concept of APE and Lorenz's APE, by regarding the latter as being a particular mathematical formalisation of the former pertaining only to purely adiabatic processes. The terms "APE" and "APE theory" are therefore used in this paper as referring to the most general concepts, thus encompassing not only Lorenz's APE and Lorenz's APE theory, but also the related concepts of exergy, which measures available potential energy relative to an isothermal reference state, e.g., Tailleux (2013) for a review, as well as attempts to incorporate momentum constraints in Lorenz's APE theory, e.g., Shepherd (1993); Codoban \& Shepherd (2003).

Although Lorenz's APE was initially formulated as a globally defined quantity by Margules (1903) and Lorenz (1955) (which Margules called the available kinetic energy), the possibility to construct it from a local principle has been known for over 30 years, e.g., Andrews (1981); Holliday \& McIntyre (1981); Shepherd (1993); Scotti et al (2006); Lamb (2007); Roullet \& Klein (2009); Molemaker et McWilliams (2010); Winters \& Barkan (2013), see Tailleux (2013) for a recent review. The local and global APE frameworks differ conceptually, however. Although the integral (1.1) is positive definite by construction, the integrand pe $-\mathrm{pe}_{r}^{*}$ can take on both signs. Lorenz's APE has been In contrast, the APE density $\mathcal{E}_{a}\left(\rho, \rho_{r}\right)$ (originally simply referred to as potential energy density by Andrews (1981) and Holliday \& McIntyre (1981)) is a local quantity that is positive definite at all points; moreover, it differs from Lorenz's APE in that it does not require the reference state to be an adiabatic re-arrangement of the actual state (see also Andrews (1981); Scotti et al (2006); Roullet \& Klein (2009)), and is therefore best seen as an energy functional operating on two density fields, the actual density field $\rho$ and the reference density fields $\rho_{r}$. As shown by Andrews (1981), the volume-integrated APE density $\mathcal{A}\left(\rho, \rho_{r}\right)$ generally exceeds Lorenz's APE, mathematically:

$$
\mathcal{A}\left(\rho, \rho_{r}\right) \geqslant \mathrm{APE}_{\text {Lorenz }},
$$

and coincides with Lorenz's APE only when the reference state coincides with Lorenz's reference state. In contrast to Lorenz's APE, the APE density is more naturally interpreted as the work against buoyancy forces that a parcel needs to perform to move from a notional reference position to its actual position, so that $\mathcal{A}\left(\rho, \rho_{r}\right)$ represents the overall work needed to construct the actual state from the reference state. The global and local APE frameworks have therefore different emphasis. The global APE budget is conceptually important for providing a way to define and quantify the power input due to boundary fluxes of buoyancy, which is lacking in traditional potential energy budgets.

The main purpose of this paper is to extend and illustrate the above ideas for a mul- 
Available Potential Energy density in nonlinear multicomponent Boussinesq fluid 3

ticomponent Boussinesq fluid with an arbitrary nonlinear equation of state, which as far as we know has never been considered previously. A strong incentive for the present work is the recent increased interest in using Lorenz (1955) exact APE theory for studying turbulent mixing in stratified fluids, e.g., Winters et al (1995) and ocean energetics, e.g., Hughes et al. (2009); Tailleux \& Rouleau (2010); Scotti \& White (2011). The present general framework should allow one to extend the latter studies - all restricted to the case of an idealised Boussinesq fluid with a linear equation of state - to more realistic settings.

Some clarification about terminology may be helpful to avoid confusion, as some readers may object against using the term "APE density" to refer to the quantity $\mathcal{E}_{a}\left(\rho, \rho_{r}\right)$ for reference density profiles $\rho_{r}$ other than Lorenz's adiabatically re-arranged density profile (or close approximate to it), on the grounds that term "APE" should be reserved to Lorenz's construct. We justify our use of terminology by the fact that APE was originally defined as the general concept concerned with identifying the part of the potential energy that can be converted into kinetic energy regardless of circumstances; in contrast, Lorenz's APE appears to represent one particular theoretical attempt at quantifying the concept APE in the particular context of purely adiabatic processes. Likewise, we use the term "APE density" as the local counterpart of the general concept of APE, not as the local counterpart of Lorenz's APE, and we hence regard the quantity $\mathcal{E}_{a}\left(\rho, \rho_{r}\right)$ as a particular theoretical attempt at quantifying the general local concept of APE, which may or may not be useful depending on how $\rho_{r}$ is chosen. As mentioned above, the quantity $\mathcal{E}_{a}\left(\rho, \rho_{r}\right)$ is conceptually identical to Andrews (1981) and Holliday \& McIntyre (1981) "potential energy density"; the terminology "APE density" seems to be preferable, however, since the volume integral of $\mathcal{E}_{a}$ is not equal to the potential energy of the fluid, but to the potential energy less a background potential energy, which is identical to Lorenz (1955) definition if one relaxes the constraint that the reference state be an adiabatic re-arrangement of the actual state.

The paper is organised as follows. Section 2 shows how to construct the APE density for a multicomponent Boussinesq fluid with a nonlinear equation of state, and discusses its local and global budgets. Section 3 discusses a number of theoretical properties of the APE density, including new proofs of results previously established by Andrews (1981). Section 4 illustrates the construction of the APE density for a number of different reference states, in the context of an idealised and realistic oceanic example. Section 5 summarises and discusses some implications of the present results. Appendix A establishes a useful result about the form of the buoyancy frequency in the present context.

\section{Local available energetics theory for a general Boussinesq model}

Motivated by geophysical applications, we take as our starting point the equations governing a general rotating multi-component incompressible stratified Boussinesq fluid with an arbitrary nonlinear equation of state, written under the form:

$$
\begin{aligned}
\frac{\mathrm{D} \mathbf{v}}{\mathrm{D} t}+2 \boldsymbol{\Omega} \times \mathbf{v}+\frac{1}{\rho_{0}} & \nabla P^{\prime}=b \nabla \Phi+\nu \nabla^{2} \mathbf{v}, \\
\nabla \cdot \mathbf{v} & =0, \\
\frac{\mathrm{D} \theta}{\mathrm{D} t} & =\dot{\theta}, \\
\frac{\mathrm{D} S_{i}}{\mathrm{D} t} & =\dot{S}_{i},
\end{aligned}
$$




$$
\rho=\rho\left(S_{i}, \theta, P_{0}(Z)\right)=\rho\left(S_{i}, \theta, Z\right),
$$

where $\mathbf{v}=(u, v, w)$ is the three-dimensional velocity field, $\boldsymbol{\Omega}$ is Earth's rotation vector, $\Phi=g_{0} Z$ is the geopotential, with $g_{0}$ a constant acceleration of gravity and $Z(z)$ the geopotential height assumed to be a monotonic function of geometric height $z, \rho$ is the density and $\rho_{0}$ a constant reference value, $P^{\prime}=P-P_{0}$ is the pressure anomaly relative to the reference Boussinesq pressure $P_{0}(Z)=P_{a}-\rho_{0} g_{0} Z$, with $P_{a}$ a constant pressure, $b=-g_{0}\left(\rho-\rho_{0}\right) / \rho_{0}$ the "standard" buoyancy defined relative to $\rho_{0}$ (we subsequently use the notation $b_{R}=-g_{0}\left(\rho-\rho_{r}\right) / \rho_{0}$ to refer to the buoyancy defined relative to an arbitrary reference state $\left.\rho_{r}\right), S_{i}(i=1, \cdots, N)$ are the $N$ variables describing chemical composition (such as salt in the oceans or total specific humidity in the atmosphere), $\theta$ a materially conserved temperature variable serving as proxy for specific entropy such as potential temperature or conservative temperature, e.g., McDougall (2003) (note that IOC, SCOR \& IAPSO (2010) recommends the spelling "Conservative Temperature" with capitalised "C" and "T"), $\nu$ the dynamic viscosity, while $\dot{\theta}$ and $\dot{S}_{i}$ are representations of the diabatic and compositional effects affecting $\theta$ and $S_{i}$. For a general geopotential $\Phi$, the hydrostatic Boussinesq pressure satisfies $\partial P_{0} / \partial z=-\rho_{0} g_{0} \partial Z / \partial z$; this implies $\partial P_{0} / \partial Z=-\rho_{0} g_{0}$, which naturally makes $P_{0}$ a function of geopotential height $Z$.

\subsection{Available potential energy density and work of buoyancy forces}

While the Boussinesq equations are classically written in terms of the standard buoyancy $b=-g_{0}\left(\rho-\rho_{0}\right) / \rho_{0}$, they can equally be written in terms of the buoyancy $b_{R}$ defined relative to any arbitrary $Z$-dependent reference density profile $\rho_{r}(Z, t)$ (which one may also choose to depend on time) viz.,

$$
b_{R}\left(S_{i}, \theta, Z, t\right)=-\frac{g_{0}\left(\rho\left(S_{i}, \theta, Z\right)-\rho_{r}(Z, t)\right)}{\rho_{0}},
$$

provided that in $(2.1)$, the pressure anomaly $P^{\prime}$ be replaced by $P-P_{r}$, where $P_{r}$ is the reference pressure profile in hydrostatic equilibrium with $\rho_{r}$ at all times, viz.,

$$
\frac{\partial P_{r}}{\partial Z}=-\rho_{r}(Z, t) g_{0}
$$

Motivated by Lamb (2007) construction of APE density building upon previous work by Andrews (1981), Holliday \& McIntyre (1981) and Scotti et al (2006), we similarly define the APE density as the following work against buoyancy forces:

$$
\mathcal{E}_{a}(S, \theta, Z, t)=-\int_{Z_{r}}^{Z} b_{R}\left(S, \theta, Z^{\prime}, t\right) \mathrm{d} Z^{\prime},
$$

and verify a posteriori that the quantity thus constructed possesses the required properties, where the level $Z_{r}$ is defined at the particular level at which the in-situ density is equal to the reference density; mathematically:

$$
\rho\left(S_{i}, \theta, Z_{r}\right)=\rho_{r}\left(Z_{r}, t\right) .
$$

Following usage in studies of atmospheric convection, it seems natural to call $Z_{r}$ the level of neutral buoyancy (LNB), which is equivalent to say that it is the implicit solution of the following LNB equation:

$$
b_{R}\left(\theta, S_{i}, Z_{r}, t\right)=0 .
$$

Equation (2.10) defines $Z_{r}=Z_{r}\left(\theta, S_{i}, t\right)$ as a function of the materially conserved variables $\theta$ and $S_{i} ; Z_{r}$ may also be time dependent if $\rho_{r}$ is.

Because the above construction can be defined for an arbitrary reference state $\rho_{r}$, 
Available Potential Energy density in nonlinear multicomponent Boussinesq fluid 5

the APE density is best viewed as defining a class or family of locally defined energy quantities. For this reason, it is also useful to introduce the notation $\mathcal{E}_{a}\left(\rho, \rho_{r}\right)$ as a reminder that the concept of APE density can also be regarded as defining a functional operating on the actual and reference density fields $\rho$ and $\rho_{r}$ respectively. Likewise, we shall denote $\mathcal{A}\left(\rho, \rho_{r}\right)$ the volume-integrated APE density as also defining a globally defined functional of the actual and reference density fields.

\subsection{Physical interpretation of the APE density}

In order to reveal the physical nature of the APE density, it is useful to re-interpret (2.8) in terms of changes in gravitational potential energy, internal energy, and work against the background pressure gradient. To show that such an interpretation applies to (2.8), let us first rewrite the change in specific gravitational potential energy $\Delta$ gpe (in units of $\mathrm{J} . \mathrm{kg}^{-1}=\mathrm{m}^{2} . \mathrm{s}^{-2}$ ) for a parcel moving from $Z_{r}$ to $Z$ as follows:

$$
\begin{gathered}
\rho_{0} \Delta \text { gpe }=\rho\left(S_{i}, \theta, Z\right) g_{0} Z-\rho\left(S_{i}, \theta, Z_{r}\right) g_{0} Z_{r}=\int_{Z_{r}}^{Z} g_{0} \frac{\partial}{\partial Z^{\prime}}\left[Z^{\prime} \rho\left(S_{i}, \theta, Z^{\prime}\right)\right] \mathrm{d} Z^{\prime} \\
=\int_{Z_{r}}^{Z} g_{0} \rho\left(S_{i}, \theta, Z^{\prime}\right) \mathrm{d} Z^{\prime}-\int_{Z_{r}}^{Z} \frac{\rho_{0} g_{0}^{2} Z^{\prime}}{c_{s}^{2}\left(S_{i}, \theta, Z^{\prime}\right)} \mathrm{d} Z^{\prime} .
\end{gathered}
$$

The last term in the r.h.s. of (2.11) represents the adiabatic change in internal energy $\Delta \mathrm{ie}_{b}$ of a fluid parcel moving from $Z_{r}$ to $Z$, since for a compressible fluid, an elementary variation of the specific internal energy $e=e\left(S_{i}, \theta, P\right)$ at constant $S_{i}$ and $\theta$ is: de $=$ $-P \mathrm{~d} v_{\text {adiabatic }}=P \mathrm{~d} P /\left(\rho^{2} c_{s}^{2}\right)$, where $c_{s}^{2}$ is the squared speed of sound. By replacing $P$ by the Boussinesq pressure $P_{0}=-\rho_{0} g_{0} Z$, as well as $\rho$ by $\rho_{0}$, the natural Boussinesq approximation of the latter is:

$$
\operatorname{die}_{b}=\frac{g_{0}^{2} Z}{c_{s}^{2}} \mathrm{~d} Z
$$

where $\mathrm{ie}_{b}$ denotes the Boussinesq approximation to specific internal energy. As a result, (2.11) can be rewritten as follows:

$$
g_{0} \int_{Z_{r}}^{Z} \rho\left(S_{i}, \theta, Z^{\prime}\right) \mathrm{d} Z^{\prime}=\rho_{0}\left(\Delta \mathrm{gpe}+\Delta \mathrm{ie}_{b}\right)=\rho_{0} \Delta \mathrm{pe}
$$

where pe $=$ gpe $+\mathrm{ie}_{b}$ denotes the Boussinesq specific potential energy, so that the APE density can be written as:

$$
\rho_{0} \mathcal{E}_{a}\left(S_{i}, \theta, Z\right)=\rho_{0}\left[\operatorname{pe}\left(S_{i}, \theta, Z\right)-\operatorname{pe}\left(S_{i}, \theta, Z_{r}\right)\right]+P_{r}(Z, t)-P_{r}\left(Z_{r}, t\right) .
$$

Equation (2.14) shows that the work against buoyancy forces done by a fluid parcel can be interpreted as the sum of two terms: the change in potential energy and the work against the background pressure gradient. Equation (2.14) is identical to (3.1) of Holliday \& McIntyre (1981) except for the added presence of internal energy in the definition of potential energy, and some differences in notations. Internal energy contributes explicitly to adiabatic changes in potential energy only when density depends on pressure, which explains why it is absent from previous expressions of APE density for a Boussinesq fluid with an equation of state linear in temperature and/or composition. Note that Boussinesq fluids admit well defined thermodynamic properties, such as internal energy, enthalpy or Gibbs function, which can be constructed explicitly as shown in Tailleux (2012). As a result, the presence of internal energy in (2.14) should not be regarded as a surprise, even though internal energy is rarely discussed in the context of Boussinesq fluids. 


\subsection{Local evolution equations for the APE density and kinetic energy}

Next, we establish that the APE density (2.8) is relevant and useful to describe potential/kinetic energy conversions. To that end, we take the substantial derivative of (2.8), which leads to:

$$
\rho_{0} \frac{D \mathcal{E}_{a}}{D t}=-\rho_{0} b_{R} W+\underbrace{\rho_{0} b_{R}\left(S, \theta, Z_{r}, t\right) \frac{D Z_{r}}{D t}}_{=0}+\rho_{0} \underbrace{\left[G_{\theta} \frac{D \theta}{D t}+G_{S_{i}} \frac{D S_{i}}{D t}+G_{t}\right]}_{G_{a}},
$$

where the term proportional to $D Z_{r} / D t$ vanishes because by definition of the LNB equation (2.10) $b_{R}\left(S_{i}, \theta, Z_{r}, t\right)=0$ if $Z_{\min }<Z_{r}<Z_{\max }$, or because $D Z_{r} / D t=0$ if $Z_{r}=Z_{\min }$ or $Z_{r}=Z_{\max }$, as we assume $Z_{\min }$ and $Z_{\max }$ to be constant. In (2.15), we defined $W=D Z / D t=\mathrm{d} Z / \mathrm{d} z w$, which reduces to the usual vertical velocity $w=D z / D t$ when $Z(z)=z$. The important terms $G_{\theta}$ and $G_{S_{i}}$ define thermodynamic efficiencies for heat and compositional sources and sinks; their expressions are:

$$
\begin{aligned}
G_{\theta} & =-\int_{Z_{r}}^{Z} \frac{\partial b_{R}}{\partial \theta}\left(\theta, S_{i}, Z^{\prime}, t\right) \mathrm{d} Z^{\prime}=\frac{g_{0}}{\rho_{0}} \int_{Z_{r}}^{Z} \frac{\partial \rho}{\partial \theta}\left(\theta, S_{i}, Z^{\prime}\right) \mathrm{d} Z^{\prime}, \\
G_{S_{i}} & =-\int_{Z_{r}}^{Z} \frac{\partial b_{R}}{\partial S_{i}}\left(\theta, S_{i}, Z^{\prime}, t\right) \mathrm{d} Z^{\prime}=\frac{g_{0}}{\rho_{0}} \int_{Z_{r}}^{Z} \frac{\partial \rho}{\partial S_{i}}\left(\theta, S_{i}, Z^{\prime}\right) \mathrm{d} Z^{\prime} .
\end{aligned}
$$

The term $G_{t}$ is only nonzero for a time-dependent reference density profile, in which case its expression is given by:

$$
G_{t}=-\int_{Z_{r}}^{Z} \frac{\partial b_{R}}{\partial t}\left(S, \theta, Z^{\prime}, t\right) \mathrm{d} Z^{\prime}=-\frac{g_{0}}{\rho_{0}} \int_{Z_{r}}^{Z} \frac{\partial \rho_{r}}{\partial t} \mathrm{~d} Z^{\prime}
$$

It is interesting to note that a term proportional to $D Z_{r} / D t$, similar to the one in (2.15), also appears in the global APE framework. In the local APE framework, this term is easily shown to vanish at each point, but in the global APE framework, it takes some effort to establish that the volume integral of a similar term is zero, e.g., Winters et al (1995); Pauluis (2007); Tailleux (2009).

Equation (2.15) states that along parcel trajectories, $\mathcal{E}_{a}$ is either converted into kinetic energy via the buoyancy flux term $b_{R} W$ or created/dissipated via the term $G_{a}$. A local evolution equation for the kinetic energy density $\mathcal{E}_{k}=\mathbf{v}^{2} / 2$, is obtained in the usual way by multiplying (2.1) by $\rho_{0} \mathbf{v}$ (with $P^{\prime}$ replaced by $P-P_{r}$ and $b$ by $b_{R}$ ), leading to:

$$
\rho_{0} \frac{D \mathcal{E}_{k}}{D t}+\nabla \cdot\left[\left(P-P_{r}\right) \mathbf{v}-\rho_{0} \nu \nabla \mathcal{E}_{k}\right)=\rho_{0} b_{R} W-\rho_{0} \varepsilon_{K}
$$

where $\rho_{0} \varepsilon_{K}=\rho_{0} \nu\left[\|\nabla u\|^{2}+\|\nabla v\|^{2}+\|\nabla w\|^{2}\right]$ is the viscous dissipation rate. As expected, the buoyancy flux term $b_{R} W$ appears with a sign opposite to that in (2.15). Summing the two equations shows that the energy quantity $\mathcal{E}_{k}+\mathcal{E}_{a}$ satisfies the following equation:

$$
\rho_{0} \frac{D\left(\mathcal{E}_{k}+\mathcal{E}_{a}\right)}{D t}+\nabla \cdot\left[\left(P-P_{r}\right) \mathbf{v}-\rho_{0} \nu \nabla \mathcal{E}_{k}\right]=\rho_{0} G_{a}-\rho_{0} \varepsilon_{K}
$$

This expression makes it clear that in the absence of diabatic and compositional effects (yielding $G_{S_{i}}=G_{\theta}=0$ ), as well as for a time-independent reference state (yielding $\left.G_{t}=0\right)$, the sum $\mathcal{E}_{k}+\mathcal{E}_{a}$ is a conservative quantity. An alternative expression for (2.20) in absence of diabatic, compositional and viscous effects is:

$$
\rho_{0} \frac{D}{D t}\left[\mathcal{E}_{k}+\mathcal{E}_{a}+\frac{P-P_{r}}{\rho_{0}}\right]=\frac{\partial\left(P-P_{r}\right)}{\partial t} .
$$


Available Potential Energy density in nonlinear multicomponent Boussinesq fluid 7

In the case where the right-hand side of (2.21) can be neglected, a Bernoulli-like theorem is obtained, stating that $\mathcal{E}_{k}+\mathcal{E}_{a}+\left(P-P_{r}\right) / \rho_{0}$ is conserved along fluid trajectories.

\subsection{Volume integrated budget of APE density}

Because the APE density $\mathcal{E}_{a}\left(\rho, \rho_{r}\right)$ is defined relative to an arbitrary reference state $\rho_{r}$, its volume-integrated budget must differ from Lorenz's APE budget, except when $\rho_{r}$ coincides with Lorenz's reference density profile $\rho_{r}^{*}$. To examine the issue, let us assume that $\theta$ and $S_{i}$ obey conservative equations of the form:

$$
\frac{D \theta}{D t}=\nabla \cdot \mathbf{F}_{\theta}, \quad \frac{D S_{i}}{D t}=\nabla \cdot \mathbf{F}_{S_{i}},
$$

and that the velocity v satisfies no-normal flux boundary conditions. Assuming a steadystate (so that both $\partial \mathcal{E}_{a} / \partial t$ and $G_{t}$ vanish), we integrate (2.15) over the fluid domain, which yields the following expression for the global buoyancy flux:

$$
\int_{V} \rho_{0} b_{R} W \mathrm{~d} V=\int_{V} \rho_{0} b W \mathrm{~d} V=\mathcal{P}_{A}-\mathcal{D}_{A}
$$

where $\mathcal{P}_{A}$ is the production term by boundary buoyancy fluxes and $\mathcal{D}_{A}$ the dissipation term by internal diffusive processes, which are respectively given by:

$$
\begin{gathered}
\mathcal{P}_{A}=\int_{\partial \mathcal{V}} \rho_{0} G_{\theta} \mathbf{F}_{\theta} \cdot \mathbf{n} \mathrm{d} S+\int_{\partial \mathcal{V}} \rho_{0} G_{S_{i}} \mathbf{F}_{S_{i}} \cdot \mathbf{n} \mathrm{d} S, \\
\mathcal{D}_{A}=\int_{V} \rho_{0}\left[\mathbf{F}_{\theta} \cdot \nabla G_{\theta}+\mathbf{F}_{S_{i}} \cdot \nabla G_{S_{i}}\right] \mathrm{d} V,
\end{gathered}
$$

where $\mathbf{n}$ is an outward unit normal vector. Physically, the global buoyancy flux (2.23) represents the net conversion between APE and kinetic energy, and its value is independent of the reference state entering the definition of buoyancy. On the other hand, both the production and dissipation terms $\mathcal{P}_{A}$ and $\mathcal{D}_{A}$ are individually sensitive to the choice of reference state, but their difference is not. In other words, the choice of a particular reference state alters the partition between the production of APE by buoyancy fluxes imparted at the boundaries and its dissipation by internal diffusive processes, but not the net APE/KE conversion.

For an idealised Boussinesq ocean with a linear equation of state, the boundary conditions for heat and salt at the surface are usually taken as:

$$
\mathbf{F}_{\theta} \cdot \mathbf{n}=\frac{Q}{\rho_{0} c_{p}}, \quad \mathbf{F}_{S} \cdot \mathbf{n}=S_{0}(E-P), \quad \text { at } \quad Z=0,
$$

where $Q$ is the net heat flux, $S_{0}$ a constant reference salinity, and $E-P$ the difference between evaporation and precipitation; $\mathcal{P}_{A}$ thus becomes:

$$
\mathcal{P}_{A}=\int_{\partial V}\left[\frac{\alpha g_{0} Z_{r}}{c_{p}} Q-\rho_{0} \beta g_{0} Z_{r} S_{0}(E-P)\right] \mathrm{d} S,
$$

where $\alpha=-\rho_{0}^{-1} \partial \rho / \partial \theta$ and $\beta=\rho_{0}^{-1} \partial \rho / \partial S$ are the thermal expansion and haline contraction coefficients respectively, defined relative to the $(\theta, S)$ variables. Since $Z_{r}<0$, only cooling $(Q<0)$ and net evaporation $(E-P>0)$ contribute to the creation of APE, so that the latter coincides with the densification of surface waters, which acts in destabilising the fluid and creating motion. The choice of a different reference state may alter $Z_{r}$ and hence the overall value of $\mathcal{P}_{A}$ but does not alter the expression (2.27) for $\mathcal{P}_{A}$. If $Z_{r}$ is only an approximation to the "true" $Z_{r}^{*}$ (to the extent that a precise definition 
of the latter can be given, perhaps in terms of Lorenz's reference state), an estimate of the error bar for $\mathcal{P}_{A}$ is:

$$
\delta \mathcal{P}_{A}=\int_{\partial V} \delta Z_{r}\left[\frac{\alpha g_{0}}{c_{p}} Q-\rho_{0} \beta g_{0} S_{0}(E-P)\right] \mathrm{d} S,
$$

with $\delta Z_{r}=Z_{r}^{*}-Z_{r}$. Error bars on current estimates of APE production in the oceans, e.g., Oort et al. (1994), are very large, but so far, they have only been derived in the context of the Lorenz's QG approximation for APE; it would be of interest to see if these could be reduced in the context of the present exact framework.

\section{Formal properties of the APE density framework}

\subsection{Positive definite character of the APE density}

Depending on the nature of the fluid considered, as well as on how the reference state is defined, the Level of Neutral Buoyancy (LNB) equation (2.10) may admit only one, several, or even no solution within the interval $\left[Z_{\min }, Z_{\max }\right]$. In most practical cases, the LNB equation usually possesses only one solution, in which case there is no ambiguity how the APE density (2.8) should be computed, but some discussion is needed in the case the LNB equation possesses multiple roots or no solution. If the LNB equation has multiple solutions, $Z_{r}$ is selected as the solution that is the closest to $Z$ satisfying $Z>Z_{r}$ if $b_{R}>0$ or $Z<Z_{r}$ if $b_{R}<0$. If no solution exists, we impose $Z_{r}=Z_{\max }$ if $b_{R}>0$ or $Z_{r}=Z_{\min }$ if $b_{R}<0$. These four different cases are illustrated in Fig. 1. This way of constructing $Z_{r}$ ensures the positive definite character of $\mathcal{E}_{a}$, since by construction $Z_{r}>Z$ if $b_{R}>0$ and $Z_{r}<Z$ if $b_{R}<0$, so that $\mathcal{E}_{a}$ vanishes only when $Z=Z_{r}$.

Another way to approach the problem is to use the definition of the parcel reference position: $\rho\left(S_{i}, \theta, Z_{r}\right)=\rho_{r}\left(Z_{r}, t\right)$ to rewrite $(2.8)$ in the following mathematically equivalent ways:

$$
\begin{gathered}
\mathcal{E}_{a}=\frac{g_{0}}{\rho_{0}} \int_{Z_{r}}^{Z}\left[\rho\left(S_{i}, \theta, Z^{\prime}\right)-\rho_{r}\left(Z^{\prime}, t\right)\right] \mathrm{d} Z^{\prime} \\
=\frac{g_{0}}{\rho_{0}} \int_{Z_{r}}^{Z}\left[\rho\left(S_{i}, \theta, Z^{\prime}\right)-\rho\left(S_{i}, \theta, Z_{r}\right)\right] \mathrm{d} Z^{\prime}-\frac{g_{0}}{\rho_{0}} \int_{Z_{r}}^{Z}\left[\rho_{r}\left(Z^{\prime}, t\right)-\rho_{r}\left(Z_{r}, t\right)\right] \mathrm{d} Z^{\prime} \\
=\frac{g_{0}}{\rho_{0}} \int_{Z_{r}}^{Z} \int_{Z_{r}}^{Z^{\prime}} \frac{\partial \rho}{\partial Z^{\prime \prime}}\left(S_{i}, \theta, Z^{\prime \prime}\right) \mathrm{d} Z^{\prime \prime} \mathrm{d} Z^{\prime}-\frac{g_{0}}{\rho_{0}} \int_{Z_{r}}^{Z} \int_{Z_{r}}^{Z^{\prime}} \frac{\partial \rho_{r}}{\partial Z^{\prime \prime}}\left(Z^{\prime \prime}, t\right) \mathrm{d} Z^{\prime \prime} \mathrm{d} Z^{\prime} \\
=-\frac{g_{0}}{\rho_{0}} \int_{Z_{r}}^{Z} \int_{Z_{r}}^{Z^{\prime}}\left[\frac{\partial \rho_{r}}{\partial Z^{\prime \prime}}\left(Z^{\prime \prime}, t\right)+\frac{\rho_{0} g_{0}}{c_{s}^{2}\left(S_{i}, \theta, Z^{\prime \prime}\right)}\right] \mathrm{d} Z^{\prime \prime} \mathrm{d} Z^{\prime} .
\end{gathered}
$$

The first equality is obtained by subtracting $\rho\left(S_{i}, \theta, Z_{r}\right)$ from the first integral and adding $\rho_{r}\left(Z_{r}, t\right)$ to the second integral; the second equality is obtained by using the result that any function can be written as the integral of its derivative; the third equality makes use of the result $\partial \rho\left(S_{i}, \theta, Z\right) / \partial Z=-\rho_{0} g_{0} / c_{s}^{2}\left(S_{i}, \theta, Z\right)$. Note that by definition of the geopotential, we have $Z=Z(z)$ and $Z_{r}=Z\left(z_{r}\right)$, so that at leading order $Z-Z_{r}=$ $Z(z)-Z\left(z_{r}\right) \approx \mathrm{d} Z / \mathrm{d} z\left(z_{r}\right)\left(z-z_{r}\right)$. As shown in appendix A, the term within square brackets is proportional to the squared buoyancy frequency defined from the reference density profile $\rho_{r}$. This makes it clear that $\mathcal{E}_{a}$ must be positive if $N_{r}^{2}$ is positive. In the limit of small amplitude vertical displacements, (3.1) reduces at leading order to:

$$
\mathcal{E}_{a} \approx-\frac{g_{0}}{\rho_{0}} \int_{Z_{r}}^{Z} \int_{Z_{r}}^{Z^{\prime}}\left[\frac{\partial \rho_{r}}{\partial Z}\left(Z_{r}, t\right)+\frac{\rho_{0} g_{0}}{c_{s}^{2}\left(S_{i}, \theta, Z_{r}\right)}\right] \mathrm{d} Z^{\prime \prime} \mathrm{d} Z^{\prime} \approx N_{r}^{2}\left(S_{i}, \theta, t\right) \frac{\zeta^{2}}{2},
$$


(a)

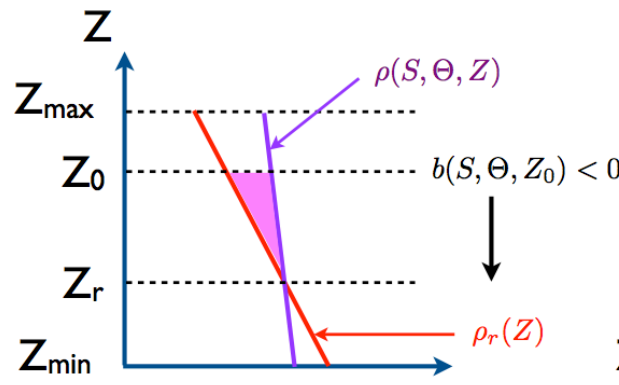

(c)

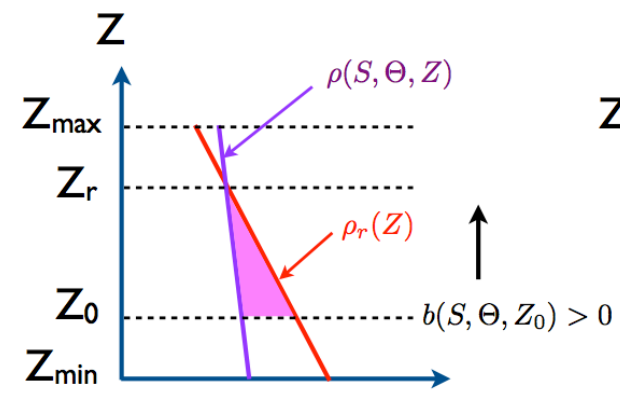

(b)

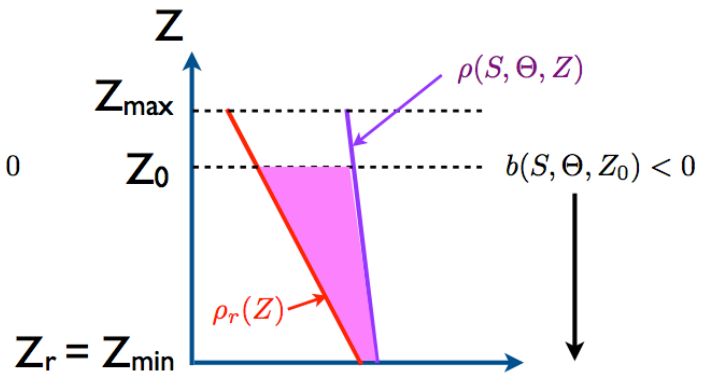

(d)

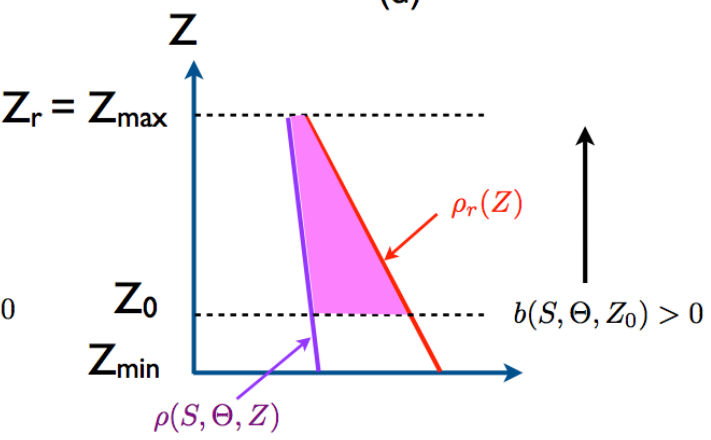

FIGURE 1. Schematics illustrating the construction of a parcel reference position in the 4 different possible cases described in the text. The sign of the local buoyancy $b$ determines whether to look for the LNB above $(b>0)$ or below $(b<0)$ the parcel current level located at $Z=Z_{0}$. The parcel reference position is chosen as the LNB that is the closest to the current level's position located if one or several such LNB exist, or at one of the fluid boundaries otherwise.

which is the well known formula for the APE density of small amplitude internal gravity waves. The same result pertains to Holliday \& McIntyre (1981)'s previous formula, which helps clarify the connection between the present approach and previous ones. Note here that the expression for the buoyancy frequency derived in Appendix A represents the period of small oscillations around the reference state only when the vertical pressure gradient term can be neglected relative to the buoyancy force in the vertical momentum equation, which is true only when the reference state is close to Lorenz's reference state. The physical meaning of $N_{r}^{2}$ for other types of reference state is less clear.

The consideration of an LNB equation with no or multiple solutions appears to be new, for previous studies, e.g., Andrews (1981); Scotti et al (2006); Roullet \& Klein (2009) all assumed that the LNB equation had to possess at least one solution for the construction of the APE density to be possible. In order to illustrate the feasibility of constructing the APE density in the case where the LNB equation hardly possesses any solution, we consider in section 4.1 the special case of a constant density reference profile $\rho_{r}(Z)=\rho_{0}$, for which $Z_{r}$ is equal to either $Z_{\min }$ or $Z_{\max }$ nearly everywhere. 
3.2. Link between the volume-integrated APE density and Lorenz's globally-defined APE

To clarify the link between the present local APE density framework and Lorenz (1955) original global concept, it is useful to integrate (2.14), which leads to:

$$
\mathcal{A}\left(\rho, \rho_{r}\right)=\mathrm{PE}-\mathrm{PE}_{r}+\int_{V}\left(P_{r}(Z, t)-P_{r}\left(Z_{r}, t\right)\right) \mathrm{d} V .
$$

The term $\mathrm{PE}-\mathrm{PE}_{r}$ in the right-hand side of (3.3) represents the difference in potential energies between the actual and reference states; $\mathcal{A}\left(\rho, \rho_{r}\right)$ is therefore identical to Lorenz (1955) global APE definition but for the presence of the pressure terms in the r.h.s. of (3.3). While the latter are non zero in general, they can be shown to vanish when the reference state $\rho_{r}$ coincides with Lorenz's adiabatically re-arranged reference state $\rho_{r}^{*}$. Indeed, assuming that Lorenz's reference state can be reached by means of a physically realisable process defining a volume-conserving map $\mathbf{x}_{r}^{*}=\mathbf{x}_{r}^{*}(\mathbf{x})$ of Jacobian $\partial\left(x_{r}^{*}, y_{r}^{*}, z_{r}^{*}\right) / \partial(x, y, z)=1$ between the actual and reference states, some standard algebra immediately yields:

$$
\int_{V} P_{r}\left(Z_{r}^{*}, t\right) \mathrm{d} V=\int_{V^{*}} P_{r}\left(Z\left(z_{r}^{*}\right), t\right) \mathrm{d} V_{r}^{*}=\int_{V} P_{r}(Z(z), t) \mathrm{d} V .
$$

The first equality follows from rewriting the integral using the change of variables $\mathbf{x}=$ $\mathbf{x}\left(\mathbf{x}_{r}^{*}\right)$, using the result that $\mathrm{d} V=\partial(\mathbf{x}) / \partial\left(\mathbf{x}_{r}^{*}\right) \mathrm{d} V_{r}^{*}=\mathrm{d} V_{r}^{*}$; the second equality follows from that $z_{r}^{*}$ and $\mathrm{d} V_{r}^{*}=\mathrm{d} x_{r}^{*} \mathrm{~d} y_{r}^{*} \mathrm{~d} z_{r}^{*}$ are dummy variables of integration, which can be replaced respectively by $z$ and $\mathrm{d} V=\mathrm{d} x \mathrm{dyd} z$ under the integral sign. Equation (3.4) implies that the pressure terms in (3.3) vanishes identically when $\rho_{r}=\rho_{r}^{*}$, and hence that:

$$
\mathcal{A}\left(\rho, \rho_{r}^{*}\right)=\mathrm{PE}-\mathrm{PE}_{r}^{*}=\mathrm{APE}_{\text {Lorenz }}
$$

which is the desired result. Alternative (but often more involved) demonstrations of the above result are given by Andrews (1981) and Shepherd (1993) for the compressible case, and by Holliday \& McIntyre (1981), Lamb (2008), Roullet \& Klein (2009) and Winters \& Barkan (2013) for a Boussinesq fluid with a linear equation of state. It is important to note that Lorenz's reference state is a special case, in the sense that the pressure terms in (3.3) may a priori become significant and sometimes dominant for reference states that are not adiabatic re-arrangements of the actual state.

\subsection{Extremal property of Lorenz's reference state in the space of all reference states}

An important result established by Andrews (1981) is that for a compressible fluid, Lorenz's reference state $\rho_{r}^{*}$ is the particular reference state that minimises the functional $\mathcal{A}\left(\rho, \rho_{r}\right)$ in the space of all reference states. Andrews (1981)'s proof is based on the consideration of a notional initial value problem whereby some external agent is assumed to drive the fluid from its rest state to its actual state by means of a purely adiabatic process. A much simpler proof, however, consists in adding and subtracting the terms $\mathrm{PE}_{r}^{*}$ and $P_{r}\left(Z_{r}^{*}\right)$ in (3.3), and forming the following combinations of terms:

$$
\begin{gathered}
\mathcal{A}\left(\rho, \rho_{r}\right)=\mathrm{PE}-\mathrm{PE}_{r}+\int_{V}\left[P_{r}(Z)-P_{r}\left(Z_{r}\right)\right] \mathrm{d} V \\
=\underbrace{\mathrm{PE}-\mathrm{PE}_{r}^{*}}_{\mathrm{APE}_{\text {Lorenz }}}+\underbrace{\mathrm{PE}_{r}^{*}-\mathrm{PE}_{r}+\int_{V}\left[P_{r}\left(Z_{r}^{*}\right)-P_{r}\left(Z_{r}\right)\right] \mathrm{d} V}_{=\mathcal{A}\left(\rho_{r}^{*}, \rho_{r}\right) \geqslant 0}+\underbrace{\int_{V}\left[P_{r}(Z)-P_{r}\left(Z_{r}^{*}\right)\right] \mathrm{d} V}_{=0} .
\end{gathered}
$$

The first term in the second line of (3.6) is simply $\mathrm{APE}_{\text {Lorenz }}$; the second term can be recognised as the quantity $\mathcal{A}\left(\rho_{r}^{*}, \rho_{r}\right)$, which measures the (fictitious) APE of Lorenz's 
Available Potential Energy density in nonlinear multicomponent Boussinesq fluid 11

reference state relative to the arbitrary reference state $\rho_{r}$, and which is positive by construction; the third term vanishes on account of the result derived in section 3.2. This clearly establishes that

$$
\int_{V} \rho_{0} \mathcal{E}_{a} \mathrm{~d} V=\mathcal{A}\left(\rho, \rho_{r}\right)=\mathcal{A}\left(\rho, \rho_{r}^{*}\right)+\mathcal{A}\left(\rho_{r}^{*}, \rho_{r}\right) \geqslant \mathrm{APE}_{\text {Lorenz }},
$$

which is the sought-for result.

\section{Discussion of some practical points on concrete examples}

4.1. Construction of the APE density for a constant reference density profile

To illustrate the feasibility of constructing the APE density for reference states whose LNB equation 2.9 possesses almost no solutions, we focus on the particular case of a constant density reference state $\rho_{r}(Z, t)=\rho_{0}=$ constant, for which the reference position $Z_{r}$ of a fluid parcel can take only three different possible values, namely $Z_{\text {min }}$, $Z$ or $Z_{\max }$, depending on whether $\rho>\rho_{0}, \rho=\rho_{0}$ or $\rho<\rho_{0}$ respectively (assuming that $\left.\rho_{\text {min }}<\rho_{0}<\rho_{\text {max }}\right)$; the corresponding expression for $\mathcal{E}_{a}\left(\rho, \rho_{0}\right)$ is thus given by:

$$
\mathcal{E}_{a}\left(\rho, \rho_{0}\right)=\left\{\begin{array}{cc}
-\int_{Z_{\max }}^{Z} b\left(S, \theta, Z^{\prime}\right) \mathrm{d} Z^{\prime}, & \rho<\rho_{0} \\
-\int_{Z_{\min }}^{Z} b\left(S, \theta, Z^{\prime}\right) \mathrm{d} Z^{\prime}, & \rho>\rho_{0} \\
0, & \rho=\rho_{0}
\end{array},\right.
$$

where $b=-g_{0}\left(\rho-\rho_{0}\right) / \rho_{0}$. Physically, the constant density $\rho_{0}$ categorises the water masses into two categories: "dense" and "light", and causes $\mathcal{E}_{a}\left(\rho, \rho_{0}\right)$ to be discontinuous across the interface $z=-h_{0}(x, y, t)$ along which $\rho(x, y, z, t)=\rho_{0}$, which separates the light from the dense waters.

It is of interest to note that the volume-integrated budget of $\mathcal{E}_{a}\left(\rho, \rho_{0}\right)$ admits a nontrivial production term by surface buoyancy fluxes. Thus, for the idealised oceanic example discussed above, assumed to be such that $Z_{\max }=0$ at the ocean surface and $Z_{\min }=-H_{\max }$, with $H_{\max }$ the maximum ocean depth, one obtains:

$$
\mathcal{P}_{A}\left(\rho_{0}\right)=-g_{0} H_{\max } \int_{\mathcal{S}\left(\rho>\rho_{0}\right)}\left[\frac{\alpha Q}{c_{p}}-\rho_{0} \beta S_{0}(E-P)\right] \mathrm{d} \mathcal{S},
$$

where the condition $\mathcal{S}\left(\rho>\rho_{0}\right)$ means that the surface integral is restricted to "dense" fluid parcels only. (4.2) defines $\mathcal{P}_{A}$ as a function of $\rho_{0}$; clearly $\mathcal{P}_{A}=0$ for both $\rho_{0}=\rho_{\text {min }}$ and $\rho_{0}=\rho_{\max }$, in the first case because all surface points are excluded from the integral, and in the second case, because the integral of the surface buoyancy fluxes vanishes by the steady-state assumption. As a result, $\mathcal{P}_{A}$ should be positive and reach a maximum for $\rho_{0}$ somewhere in between $\rho_{\min }$ and $\rho_{\max }$, provided that the surface buoyancy fluxes be on average negative where surface parcels are the densest, as is usually the case in the oceans. In the particular case $\rho_{0}=\rho_{\max }$, the reference position for all parcels is at the surface, and $\mathcal{E}_{a}\left(\rho, \rho_{0}\right)$ then coincides with the quantity called "effective potential energy" by Nycander (2010) and "dynamic enthalpy" by Young (2010) (note, however, that neither Young nor Nycander impose any condition on $\rho_{0}$ ), whose volume-integrated budget lacks any explicit production term by surface buoyancy fluxes.

\subsection{APE densities for a realistic ocean example}

Some of the practical issues involved in applying the above ideas to concrete problems are now illustrated for a realistic oceanic example by showing how to construct the APE density for Lorenz's reference state, as well as for the horizontal-mean density 
profile reference state. Consideration of the horizontal mean density is motivated by the fact that it is commonly used as a proxy for Lorenz's reference state in the context of the QG approximation, e.g. Von Storch et al. (2012), so it is of interest to see more precisely how the APE densities for the two kind of reference states compare precisely in the context of the exact APE density framework. To that end, we use climatological temperature and salinity fields from the World Ocean Atlas 2009, e.g., Locarnini et al (2010) and Antonov et al (2010) (The data are freely accessible at: http : //www.nodc.noaa.gov/OC5/WOA09/netcdf_data.html). While a full analysis is planned for submission to a specialised oceanographic journal, our aim here is less ambitious and only intended at the following: First, demonstrate the feasibility of computing the APE density based on the two types of reference states, and evaluate to what extent the results based on the horizontal mean density field compare with those based on the adiabatically sorted reference state. Second, show that multiple solutions of the LNB equation only occur in a very tiny region of the potential temperature/salinity space, and hence that for practical purposes, available energetics in the (present-day) ocean appears to be well-defined despite the binary nature of the fluid and the nonlinearities of the equation of state. It is important to recognise, however, that the same results do not necessarily hold for other types of multicomponent fluids; these remain to be studied on a case-by-case basis.

\subsubsection{Methodological issues}

In order to construct the APE density $\mathcal{E}_{a}(\rho, \bar{\rho})$ based on the horizontal-mean density profile $\bar{\rho}(Z)$, we first computed the in-situ density $\rho(S, \theta, Z)$ using Jackett et al (2006) equation of state, which is written in terms of potential temperature $\theta$, practical salinity $S$, and gauge pressure $P$ (i.e., the total pressure minus the standard atmospheric pressure). The WOA2009 climatological temperature and salinity fields are specified on a $1^{\circ} \times 1^{\circ}$ grid on the horizontal, and 33 standard depth levels $Z_{k}$, going from the surface $Z_{1}=0 \mathrm{~m}$ to $Z_{33}=-5500 \mathrm{~m}$. Each depth level was converted to Boussinesq pressure levels $P_{k}=-\rho_{0} g_{0} Z_{k}$, using $\rho_{0}=1027 \mathrm{~kg} . \mathrm{m}^{-3}$ and $g_{0}=9.81 \mathrm{~m} . \mathrm{s}^{-2}$. The horizontal mean density field was then computed at each of the standard depth level, yielding a discretisation of the horizontal mean density $\bar{\rho}_{k}, k=1, \cdots 33$. Solving the LNB equation $\rho(S, \theta, Z)=\bar{\rho}(Z)$ at each grid point requires a continuous description of $\bar{\rho}(Z)$, which was obtained from the standard Matlab interpolation routine. Such an interpolation function was used to solve the LNB equation at each grid point, following the methodology illustrated in the schematics of Fig. 1 . Once the LNB was found, $\mathcal{E}_{a}(\rho, \bar{\rho})$ was estimated at each grid point of the WOA2009 dataset by integrating (2.8) using a standard second order trapezoidal scheme.

A different construction is needed to obtain the APE density $\mathcal{E}_{a}\left(\rho, \rho_{r}^{*}\right)$ for Lorenz's adiabatically re-arranged reference state $\rho_{r}^{*}$, since the LNB is usually obtained from a sorting procedure of the kind described by Huang (2005), which then determines the reference density profile $\rho_{r}^{*}(Z)$. On a personal laptop, the implementation of Huang (2005)'s sorting algorithm can take up to a few hours depending on how many target depths are used; for 100 target depths, which appear to be accurate enough, about an hour was necessary to do the computation. For the WOA2009 database, as well as for other oceanographic datasets, we found that $\bar{\rho}(Z)$ and $\rho_{r}^{*}(Z)$ were in general in good agreement with each other, except near the ocean bottom and surface, as can be seen in the left panel of Fig. 2 . 
Available Potential Energy density in nonlinear multicomponent Boussinesq fluid 13
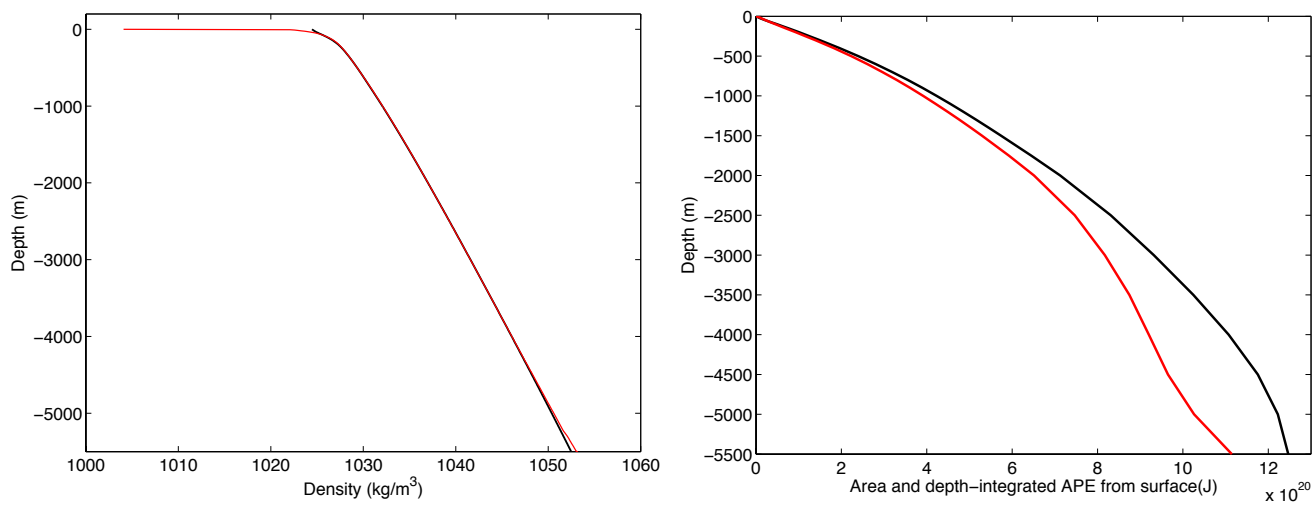

Figure 2. (Left panel) Comparison of the horizontal mean density (black line) and of the adiabatically sorted reference density based on Huang (2005) method (red line). (Right panel) Comparison of the vertical integral between the surface and a depth $Z$ of the area-integrated APE density for the two different density profiles.

\subsubsection{Cursory comparison of the two APE theories}

Fig. 2 (right panel) depicts the vertical integral between the surface and some level $\mathrm{Z}$ of the area-integrated APE density as a function of depth for the two density profiles. This shows that values pertaining to the horizontal mean density field systematically overestimate those based on Lorenz's reference state, which is expected from the result of Section 3.3, but the two curves are nevertheless of comparable magnitude (at worst differing by 10 to 20 percent from each other, which can be considered reasonable, as error bars in realistic energetics studies may often be quite large). The differences are further illustrated in Fig. 3, with a comparison of the decimal logarithm of the zonally-averaged APE density (top panels) and zonally averaged level of neutral buoyancy (bottom panels), as functions of depth and latitude. Again, there is a remarkable agreement in the structures predicted by both density profiles, which suggests that the APE density based on the horizontal mean density field might be regarded as a reasonable approximation to the APE density based on Lorenz's reference state (although further work is required to provide quantitative error bars).

\subsubsection{Uniqueness issues}

We analysed the roots of the LNB equation (2.10) in the case where $\rho_{r}(Z)=\bar{\rho}(Z)$, and usually found only one solution except in a tiny region of potential temperature/salinity space found to possess two solutions: a shallow and a deep one, illustrated respectively in the left and right panels of Fig. 4 (no solutions to the LNB equation exists in the white regions of the left panel of Fig. 4). We estimated the squared buoyancy frequency $N_{r}^{2}$ for the two types of solutions using (A 6) of Appendix A) (not shown), and found that only the shallow solution depicted in the left panel of Fig. 4 had a positive $N_{r}^{2}$. There is therefore only one admissible statically stable solution in the present case.

\section{Discussion}

The interpretation of the APE density in terms of work against buoyancy forces, e.g., Andrews (1981); Holliday \& McIntyre (1981); Lamb (2007), was shown here to generalise to a multicomponent Boussinesq fluid with a nonlinear equation of state, and to allow for a simpler and physically more intuitive construction than that based on Shepherd (1993) and Bannon (2003)'s Hamiltonian theory. As noted by previous authors, 
(a)

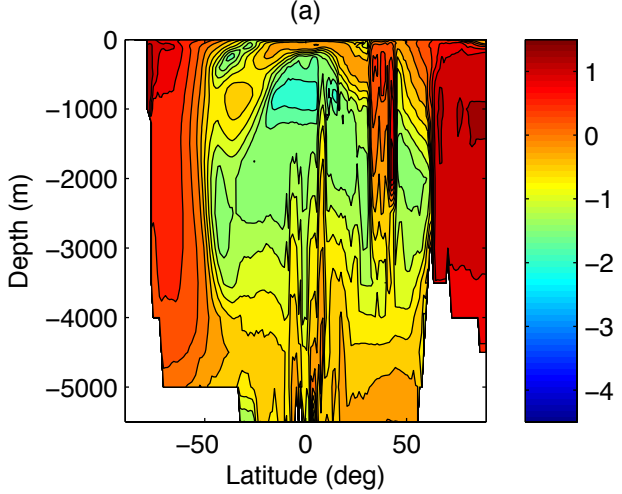

(c)

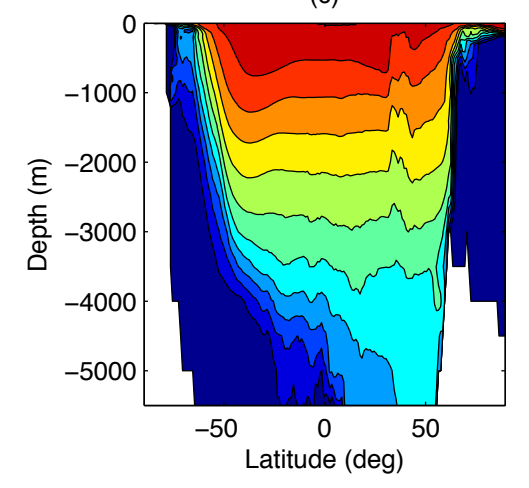

(b)
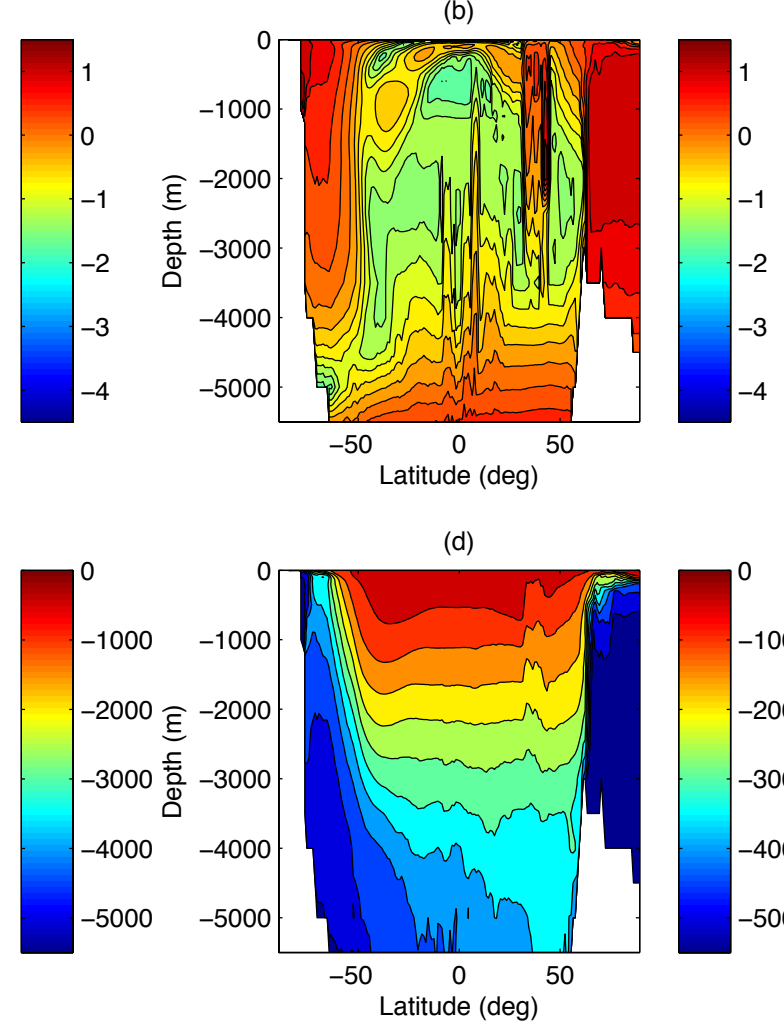

(d)

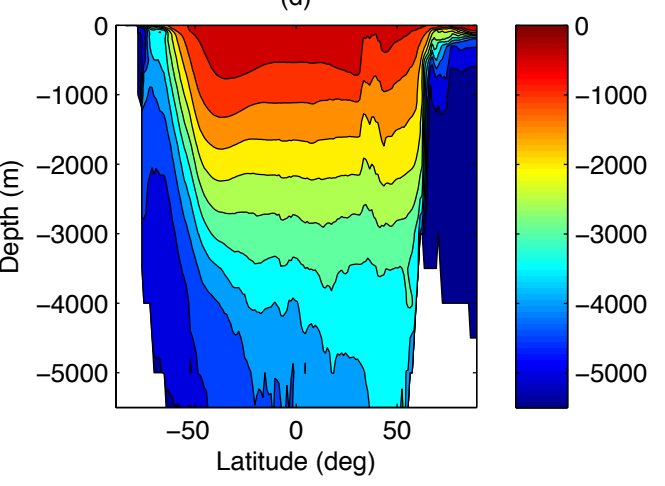

Figure 3. Decimal logarithm of the zonally-averaged APE density (expressed in $\mathrm{J}_{\mathrm{kg}}{ }^{-1}$ ) (top panels) and zonal average of the parcels' reference position (bottom panels) based on the horizontal mean density field reference state (left panels) and adiabatically sorted reference state (right panels).

the APE density $\mathcal{E}_{a}\left(\rho, \rho_{r}\right)$ and its volume integral $\mathcal{A}\left(\rho, \rho_{r}\right)$ define locally and globally defined energy functionals operating on the actual and reference density fields $\rho$ and $\rho_{r}$, with Lorenz's adiabatically re-arranged reference state $\rho_{r}^{*}$ playing a special role as the particular reference state minimising $\mathcal{A}\left(\rho, \rho_{r}\right)$ in the space of all possible reference states. Although the possibility to construct the APE density based on an arbitrary reference state has been recognised for over 30 years, e.g., Andrews (1981); Scotti et al (2006); Roullet \& Klein (2009), it has received little attention so far. This result is potentially more important than previously assumed, however, because we note that the construction allows in principle for the use of "mean" reference density field $\langle\rho\rangle$ (defined for some averaging operator $\langle$.$\rangle ) varying not only vertically, but horizontally as well, leading to$ the following APE density:

$$
\mathcal{E}_{a}=\frac{g_{0}}{\rho_{0}} \int_{Z_{r}}^{Z}\left(\rho\left(S, \theta, Z^{\prime}\right)-\langle\rho\rangle\left(x, y, Z^{\prime}, t\right)\right) \mathrm{d} Z^{\prime},
$$

with $Z_{r}$ being the solution of $\rho\left(S, \theta, Z_{r}\right)=\langle\rho\rangle\left(x, y, Z_{r}\right)$. In the small amplitude limit, $\mathcal{E}_{a, \text { small }} \approx\langle N\rangle^{2} \zeta^{2} / 2$, which is similar to (3.1) derived above, except that it now involves a locally defined $\langle N\rangle^{2}$, rather than the squared buoyancy frequency of a reference state of rest; (5.1) is therefore similar to the "eddy" APE density recently introduced in Roullet et al. (2013) discussion of the energetics of eddy motions in ARGO floats data. The study 

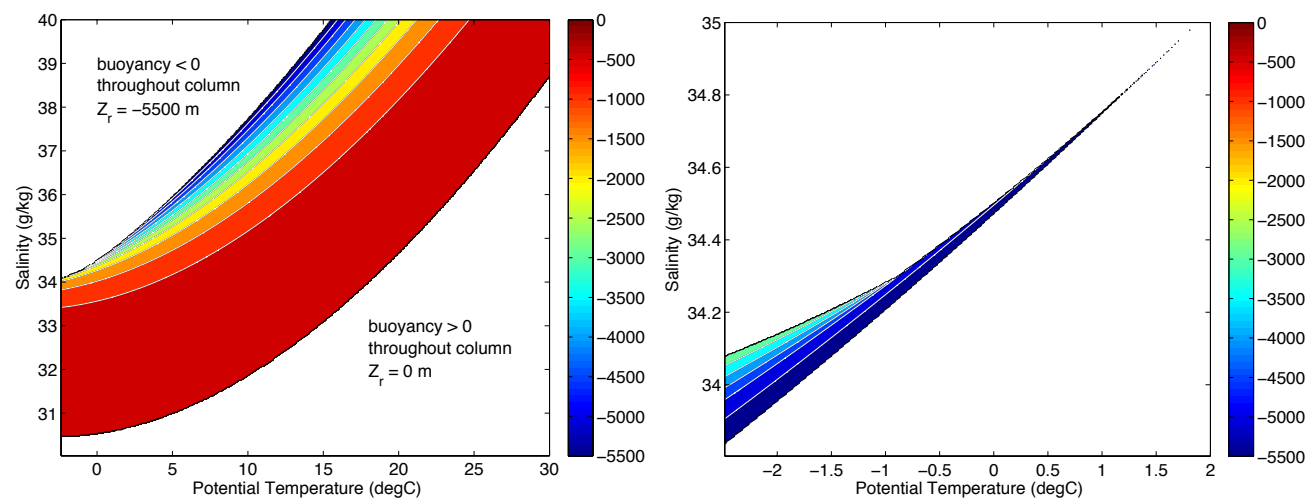

Figure 4. (Left panel) Statically stable (shallow) solution $Z_{r}(\theta, S)$ of the LNB equation $\rho_{r}\left(Z_{r}\right)=\bar{\rho}\left(Z_{r}\right)$. (Right panel) Statically unstable (deep) solution $Z_{r}(\theta, S)$ of the LNB equation (note the zoom on the tiny region of $(\theta, S)$-space where this solution exists). There are no solutions to the LNB equation in the white regions of the left panel, which are regions where the buoyancy is either strictly positive or strictly negative.

of (5.1) is beyond the scope of this paper, however, because its local evolution equation is significantly more complex, and therefore warrants a separate treatment. We regard, however, our demonstration of the feasibility of constructing the APE density for $\rho_{r}=\bar{\rho}$ and $\rho_{r}=\rho_{0}$, which are not adiabatic re-arrangements of the actual state, as a useful stepping stone towards addressing the case of a reference state varying in all three spatial directions. Note that in contrast to Lorenz (1955) QG approximation to APE, which also relies on $\bar{\rho}$ and is a prior $i$ valid only for small amplitude displacements, our construction of APE discussed in section 4 is valid for arbitrary large vertical displacements. (This is not to say that $\mathcal{E}_{a}(\rho, \bar{\rho})$ is necessarily a good approximate of $\mathcal{E}_{a}\left(\rho, \rho_{r}^{*}\right)$; this is the case only when $\bar{\rho}$ is a good approximate of $\rho_{r}^{*}$, which in the ocean is true only away from the surface and bottom).

Interestingly, the APE densities based on $\bar{\rho}$ and $\rho_{0}$ are closely related to the two following quantities:

$$
\begin{gathered}
h=-\int_{0}^{Z} b\left(S, \theta, Z^{\prime}\right) \mathrm{d} Z^{\prime}, \\
\operatorname{DPE}=h^{\prime}=h-\bar{h}=\int_{0}^{Z} \frac{g_{0}(\rho-\bar{\rho})}{\rho_{0}} \mathrm{~d} Z^{\prime}=-\int_{0}^{Z}(b-\bar{b}) \mathrm{d} Z^{\prime} .
\end{gathered}
$$

The first one, defined by (5.2), is called "dynamic enthalpy" by Young (2010) and "effective potential energy" by Nycander (2010), while the second one, defined by (5.3), is called "dynamic potential energy (DPE)" by Roquet (2013). Both $h$ and $h^{\prime}$ represent the notional work against buoyancy forces that a parcel would have to perform to move from the ocean surface to its actual position, for two different definitions of buoyancy: the standard buoyancy $b=-g_{0}\left(\rho-\rho_{0}\right) / \rho_{0}$ and the buoyancy $b=-g_{0}(\rho-\bar{\rho}) / \rho_{0}$. Equations (5.2) and (5.3) are nearly identical to their APE density counterparts but for their bounds of integration starting at the ocean surface rather than at the level of neutral buoyancy. This apparent innocuous difference has a dramatic impact on the formal properties of $h$ and $h^{\prime}$ relative to their APE density counterparts. The most obvious disadvantages of $h$ and $h^{\prime}$ are their lack of positive definiteness, which makes the problem of partitioning them into mean and eddy components ill defined, and their lack of any explicit production term by surface buoyancy fluxes, which makes it difficult to quantitatively discuss the role of the latter in powering the ocean circulation. Incidentally, $h$ and $h^{\prime}$ were proposed as 
alternative to APE for discussing ocean energetics, but the present results suggest that this is not justified since both quantities can be transformed into physically superior APE-like quantities with little difficulty.

In the present framework, a key role is played by the LNB equation (2.10) whose solution(s) determine the reference position of the fluid parcels, and whose systematic investigation provides insights into such issues as the uniqueness of a parcel reference position or of adiabatically re-arranged reference states. Although the use of a LNB equation is implicit in Andrews (1981) and Scotti et al (2006), and therefore not entirely new, it is the first time as far as we know that it is systematically exploited to gain insights into the nature of the reference state, as well as to assign a reference position to fluid parcels for reference states that are not adiabatic re-arrangements of the actual state. The use of the LNB equation to assign a parcel reference position is in contrast to the predominant approach in the literature, where a parcel reference position is usually envisioned as the outcome of a sorting procedure of the kind described in Winters et al (1995); Huang (2005); O'Gorman (2010) in various contexts. A sorting procedure, however, can only serve to determine a parcel reference position for Lorenz's adiabatically re-arranged reference state (or rather a discrete form of it), but not for more general reference states. We argue, however, that even in the context of adiabatically rearranged reference states, there is value in divorcing the practical matter of implementing a sorting procedure from the theoretical study of the solutions of the LNB equation, as the two serve different goals; notably, only the LNB equation is really suited to studying the physical properties of the reference state.

Finally, the present results suggest that the volume-integrated APE density is best viewed as being the sum of the following three terms:

$$
\mathcal{A}\left(\rho, \rho_{r}\right)=\mathrm{GPE}-\mathrm{GPE}_{r}+\mathrm{IE}-\mathrm{IE}_{r}+\text { Pressure Work, }
$$

of which Lorenz (1955)'s partition of APE into gravitational and internal energy components APE $=$ AGPE + AIE appears to be a special case. From a practical viewpoint, it is important to realise that as discussed by Winters \& Barkan (2013), the APE density $\mathcal{E}_{a}\left(\rho, \rho_{r}\right)$ is in general several orders of magnitude smaller than its individual constituents $\Delta$ pe and the pressure work taken separately; the APE density and its volume integral are therefore relatively small residuals of differences between much larger terms. From a numerical viewpoint, this indicates an ill posed problem, and suggests that the numerical estimation of AGPE, AIE and APE for Lorenz's reference state might be sensitive to small errors in the determination of the adiabatically sorted reference state. Thus, although the volume integral of the pressure work is supposed to vanish for an adiabatic re-arrangement of the actual state, this might not be so for the discrete problem. This issue is important, because it raises questions about the actual accuracy of the APE estimates of Huang (2005) and O'Gorman (2010) for a realistic ocean and atmosphere respectively, which are based on Lorenz's integral definition of APE as a residual between two large terms, but for which no error bars are given From a numerical viewpoint, it seems better to compute the APE as the volume-integral of the positive definite APE density, as this entails summing up small positive terms rather than large positive and negative ones. A full discussion of this issue is beyond the scope of this paper, but should be addressed in any studies aiming at providing accurate estimates of APE and APE production in realistic settings.

Discussions with K. Oliver, Ed Butler, J. Gregory, A. Scotti, B. White, G. Roullet, J. Molemaker, J. C. McWilliams, A. Hogg, J. Saenz, S. Urakawa, M. E. McIntyre, and W. R. Young over the past few years were useful in clarifying the issues discussed. I am 
Available Potential Energy density in nonlinear multicomponent Boussinesq fluid 17

grateful to Ed Butler for supplying the sorting code adapting Huang (2005) approach. The comments by three anonymous referees helped clarify presentation and contents.

\section{Appendix A. Buoyancy frequency of small adiabatic oscillations}

The buoyancy frequency $N_{r}$, which is usually defined as the natural frequency of small adiabatic oscillations to a state of rest, is a natural selection criterion for discriminating between multiple roots of the LNB equation (2.9). To obtain an expression for $N_{r}$, we take as our starting point the vertical momentum equation, viz.,

$$
\frac{D w}{D t}+\frac{1}{\rho_{0}} \frac{\partial\left(P-P_{r}\right)}{\partial z}=-\frac{g_{0}\left(\rho-\rho_{r}\right)}{\rho_{0}} Z^{\prime}(z)
$$

where $\rho_{r}=\rho_{r}(Z)$ is independent of time for adiabatic motions. Let us denote by $\zeta=z-z_{r}$ the vertical displacement of a fluid parcel around its level of neutral buoyancy $z_{r}$, whose geopotential height is $Z_{r}=Z\left(z_{r}\right)$. Like $Z_{r}, z_{r}$ is a materially conserved quantity for a time-independent reference state; this implies $D z_{r} / D t=0$, and hence $w=D \zeta / D t$ as well as $D w / D t=D^{2} \zeta / D t^{2}$.

The density of a parcel moving adiabatically away from its level of neutral buoyancy is given at leading order by:

$$
\rho\left(S_{i}, \theta, Z\right)=\rho\left(S_{i}, \theta, Z_{r}\right)+\int_{Z_{r}}^{Z} \frac{\partial \rho}{\partial Z^{\prime}}\left(S_{i}, \theta, Z^{\prime}\right) \mathrm{d} Z^{\prime} \approx \rho\left(S_{i}, \theta, Z_{r}\right)-\frac{\rho_{0} g_{0}\left(Z-Z_{r}\right)}{c_{s}^{2}\left(S_{i}, \theta, Z_{r}\right)},
$$

where $c_{s}^{2}$ is the squared speed of sound. The restoring buoyancy force experienced by the fluid parcel at its displaced position $Z$ depends on $\rho_{r}(Z)$; at leading order, one has:

$$
\begin{gathered}
\rho_{r}(Z) \approx \rho_{r}\left(Z_{r}\right)+\frac{\mathrm{d} \rho_{r}}{\mathrm{~d} Z}\left(Z_{r}\right)\left(Z-Z_{r}\right), \\
Z-Z_{r}=Z(z)-Z\left(z_{r}\right) \approx Z^{\prime}\left(z_{r}\right)\left(z-z_{r}\right) .
\end{gathered}
$$

Using s. (A 2), (A 3) and (A 4) as well as the definition of the LNB (2.9) in the vertical momentum equation (A 1), yields, after neglecting the vertical pressure gradient term:

$$
\frac{D^{2} \zeta}{D t^{2}}+N_{r}^{2} \zeta \approx 0
$$

with

$$
N_{r}^{2}\left(S_{i}, \theta\right)=-\frac{g_{0}}{\rho_{0}}\left(Z^{\prime}\left(z_{r}\right)\right)^{2}\left[\frac{\mathrm{d} \rho_{r}}{\mathrm{~d} z}\left(Z_{r}\right)+\frac{\rho_{0} g_{0}}{c_{s}^{2}\left(S_{i}, \theta, Z_{r}\left(S_{i}, \theta\right)\right)}\right],
$$

which is the desired expression for the squared buoyancy frequency $N_{r}^{2}$. In general, $c_{s}^{2}$ and $\rho$ are functionally unrelated, and it is therefore not possible to write $N_{r}\left(S_{i}, \theta\right)$ under the form $f\left(Z_{r}\left(S_{i}, \theta\right)\right)$ for some single-valued function $f(Z)$; this implies that $N_{r}$ cannot depend on $Z$ alone, unlike $\rho_{r}$ and $P_{r}$, and must therefore be regarded as a function of all materially conserved variables $\theta$ and $S_{i}$. This is possible because for a multicomponent fluid, different parcels may have the same density while having distinct temperature and composition, owing to density compensation; in oceanography, the concept of spiciness is used to distinguish between water masses having the same density but different values of temperature/salinity.

\section{REFERENCES}

Andrews, D. G. 1981 A note on potential energy density in a stratified compressible fluid. $J$. Fluid Mech. 107, 227-236. 
Antonov, J.I., D. Seidov, T.P. Boyer, R.A. Locarnini, A.V. Mishonov, H.E. Garcia, O.K. Baranova, M.M. Zweng, \& D.R. Johnson 2010 World Ocean Atlas 2009, Volume 2: Salinity. S. Levitus, Ed. NOAA Atlas NESDIS 69, U.S. Government Printing Office, Washington, D.C., 184.

Bannon, P. 2003 Hamiltonian description of idealized binary geophysical fluids. J. Atm. Sci. 60, 2809-2819.

Codoban, S. \& Shepherd, T. G. 2003 Energetics of a symmetric circulation including momentum constraints. J. Atmos. Sci. 60, 2019-2028.

Gregory, J.M. \& Tailleux, R. 2010 Energetic analysis of simulated time-dependent changes in the Atlantic meriidonal overturning circulation in response to increasing $\mathrm{CO}_{2}$ concentration. Clim. Dyn. 37, 893-914.

Holliday, D. \& McIntyre, M. E. 1981 On potential energy density in an incompressible stratified fluid. J. Fluid Mech. 107, 221-225.

HuAng, R. X. 2005 Available potential energy in the world's oceans. J. Mar. Res. 63, 141-158.

Hughes, G.0., Hogg, A., \& Griffiths, R.W. 2009 Available potential energy and irreversible mixing in the meridional overturning circulation. J. Phys. Oceanogr. 39, 3130-3146.

IOC, SCOR \& IAPSO 2010 The international thermodynamic equation of seawater- 2010: Calculations and use of thermodynamic properties. Intergovernmental Oceanographic Commission, Manuals and Guides No 56 ed, $196 \mathrm{pp}$.

Jackett, D. R., T. J. McDougall, R. Feistel, D. G. Wright \& S. M. Griffies 2006 Algorithms for density, potential temperature, conservative temperature, and freezing temperature of seawater. J. Atmos. Oceanic Technol., 23, 1709-1728.

Kucharski, F. 1997 On the concept of exergy and available potential energy. Q. J. Roy. Met. Soc. 123, 2141-2156.

LAMB (2007) 2007 Energy and pseudoenergy flux in the internal wave field generated by tidal flow over topography. Cont. Shelf Res., 27, 1208-1232.

LAMB, K. G. (2008) 2008 On the calculation of the available potential energy of an isolated perturbation in a density stratified fluid. J. Fluid Mech. 597, 415-427.

Locarnini, R.A., A.V. Mishonov, J. I. Antonov, T.P. Boyer, H. E. Garcia, O.K. BaraNova, M.M. Zweng, \& D.R. Johnson 2010 World Ocean Atlas 2009, Volume 1: Temperature. S. Levitus, Ed. NOAA Atlas NESDIS 68, U.S. Government Printing Office, Washington, D.C., 184 pp.

Lorenz, E. N. 1955 Available potential energy and the maintenance of the general circulation. Tellus 7, 157-167.

Margules, M.1903 Über die Energie der Stürme. Jahrb. Zentralanst. Meteorol. Wien 40, 1-26.

MCDougall, T.J.2003 Potential enthalpy: A conservative oceanic variable for evaluating heat content and heat fluxes. J. Phys. Oceanogr. 33, 945-963.

Molemaker, M. J. \& McWilliams, J. C. 2010 Local balance and cross-scale flux of available potential energy. J. Fluid Mech. 645 295-314.

NyCANDER, J. 2010 Horizontal convection with a nonlinear equation of state: generalization of a theorem of Paparella and Young. Tellus, 62A, 134-137.

O'Gorman, P.A. 2010 Understanding the varied response of the extratropical storm tracks to climate change. PNAS, 107, 19,176-19,180.

Oort, A.H., Anderson, L.A., \& Peixoto, J.P. 1994 Estimates of the energy cycle of the oceans. J. Geophys. Res. 99, C4, 7665-7688.

PAuluis, O. 2007 Sources and sinks of available potential energy in a moist atmosphere. J. Atm. Sc., 64, 2627-2641.

Peixoto, J. P. \& Oort, A. H. 1992 Physics of climate. American Institute of Physics, New York. 520 pp.

Roquet, F. 2013 Dynamical potential energy: A new approach to ocean energetics. J. Phys. Oceanogr., 43, 457-476.

Roullet, G. \& Klein, P. 2009 Available potential energy diagnosis in a direct numerical simulation of rotating stratified turbulence. J. Fluid Mech. 624, 45-55.

Roullet, G., X. CApet \& G. Maze 2013 Oceanic interior mesoscale turbulence revealed by ARGO floats. Geophys. Res. Lett. submitted.

Scotti, A., R. Beardsley, \& B. Rutman 2006 On the interpretation of energy and energy 
fluxes of nonlinear internal waves: an example from Massachussetts Bay. J. Fluid Mech. 561, 103-112.

Scotti, A. \& B. White 2011 Is horizontal convection really "non-turbulent"? Geophs. Res. Lett., 38, doi:10.1029/2011GL049701.

Shepherd, T.G. 1993 A unified theory of available potential energy. Atmosphere-Ocean 31, $1-26$.

Tailleux, R. \& Grandpeix, J. Y. 2004 On the seemingly incompatible parcel and globally integrated views of the energetics of triggered atmospheric deep convection over land. $Q$. J. Roy. Met. Soc. 130, 3223-3243.

TAIlleux, R. 2009a On the energetics of stratified turbulent mixing, irreversible thermodynamics, Boussinesq models, and the ocean heat engine controversy. J. Fluid Mech. 638, 339-382.

Tailleux, R. \& Rouleau, L. 2010 The effect of mechanical stirring on horizontal convection. Tellus A 62, 138-153.

TAILleux, R. 2012 Thermodynamics/dynamics coupling in weakly compressible turbulent stratified fluids. IRSRN Thermodyn. 2012:609701.

Tailleux, R. 2013 Available potential energy and exergy in stratified fluids. Annu. Rev. Fluid Mech., 45, 35-58.

VAlLis, G.2006 Atmospheric and oceanic fluid dynamics. Cambridge University Press.

von Storch, J.-S., C. Eden, I. Fast, H. HaAk, D. Hernandez-Deckers, E. MaierReimer, J. Marotzke, \& D. Stammer 2012 An estimate of the Lorenz energy cycle for the world ocean based on the STORM/NCEP simulation. J. Phys. Oceanogr. 42, 21852205.

Winters, K. B., Lombard, P. N., And Riley, J. J., \& D’Asaro 1995 Available potential energy and mixing in density-stratified fluids. J. Fluid Mech. 289, 115-228.

Winters, K. B. \& R. BARKAn 2013 Available potential energy density for Boussinesq fluid flow. J. Fluid Mech. 714, 476-488.

Young, W. R. 2010 Dynamic enthalpy, conservative temperature, and the seawater Boussinesq approximation. J. Phys. Oceanogr., 40, 394-400. 